\title{
Dynamics of viscous fingers in rotating Hele-Shaw cells with Coriolis effects
}

\author{
Hermes Gadêlha and Nielison Brito \\ Laboratório de Física Teórica e Computacional, Departamento de Física, Universidade Federal de Pernambuco, Recife, Pernambuco \\ 50670-901 Brazil \\ José A. Miranda* \\ Department of Physics, University of Florida, P.O. Box 118440, Gainesville, Florida 32611-8440, USA
}

(Received 19 June 2006; published 17 January 2007)

\begin{abstract}
A growing number of experimental and theoretical works have been addressing various aspects of the viscous fingering formation in rotating Hele-Shaw cells. However, only a few of them consider the influence of Coriolis forces. The studies including Coriolis effects are mostly restricted to the high-viscosity-contrast limit and rely on either purely linear stability analyses or intensive numerical simulations. We approach the problem analytically and use a modified Darcy's law including the exact form of the Coriolis effects to execute a mode-coupling analysis of the system. By imposing no restrictions on the viscosity contrast $A$ (dimensionless viscosity difference) we go beyond linear stages and examine the onset of nonlinearities. Our results indicate that when Coriolis effects are taken into account, an interesting interplay between the Reynolds number Re and $A$ arises. This leads to important changes in the stability and morphological features of the emerging interfacial patterns. We contrast our mode-coupling approach with previous theoretical models proposed in the literature.
\end{abstract}

DOI: 10.1103/PhysRevE.75.016305

PACS number(s): 47.15.gp, 47.54.-r, 47.20.Ma, 47.55.D-

\section{INTRODUCTION}

The study of the evolution of hydrodynamic instabilities and pattern formation at the interface separating two fluids in a rotating Hele-Shaw cell has attracted much interest since the initial work by Schwartz [1-20]. The problem constitutes a variation of the traditional, purely viscosity-driven Saffman-Taylor instability $[21,22]$ in which the cell is rotated around a vertical axis passing through its center. As a result of the density difference between the two confined fluids, centrifugally induced interfacial deformations arise, leading to various interesting dynamical and morphological effects.

Despite the considerable number of theoretical and experimental studies of rotating Hele-Shaw cells [1-20], just a few of them [1,15-18] address the role of Coriolis forces in affecting the dynamics of the interface. On the analytical side, the structure of the fingering under Coriolis forces is largely restricted to linear stability investigations $[1,15,16]$ and mostly limited to the high-viscosity-contrast case (inviscid outer fluid). The unbounded outer fluid problem studied in Ref. [15] has been extended [16] to cover the linear stability of the two-fluid problem (fluids contained within a bounded disk), with arbitrary viscosity contrast and density ratio. These studies provide useful information about the system during the initial (purely linear) time regime. A noteworthy point originally examined in Ref. [15] refers to the way the Coriolis force is added into the governing equations for fluid flow in rotating Hele-Shaw cells: while Schwartz [1] includes it directly in a gap-averaged two-dimensional (2D) Darcy's law, Waters and Cummings [15] introduce it in a more rigorous fashion onto a 3D Navier-Stokes equation. Comparison between these two approaches at the linear level

\footnotetext{
*On leave from Departamento de Física, LFTC, UFPE, Brazil. Electronic address: jme@df.ufpe.br
}

[15] supports the generality of the Navier-Stokes firstprinciples calculation, restricting the quantitative validity of the more casual model of Ref. [1] to considerably small Reynolds numbers (relative measure of inertial and viscous forces). More advanced (fully nonlinear) stages of the interfacial evolution including Coriolis forces are also not completely understood and rely heavily on numerical simulations for immiscible [1] and miscible [17,18] flows. To this day, no attention has been paid to an analytical investigation of the dynamics that bridges these two extreme stages under the presence of Coriolis forces. Consideration of arbitrary values of the viscosity contrast has also been largely overlooked.

Recently, the interfacial instability of two viscous fluids in a rotating Hele-Shaw cell has been studied (neglecting Coriolis effects) by analytical mode-coupling approaches $[10,13]$. In particular, it has been shown that a second-order weakly nonlinear analysis is capable of accurately describing essential nonlinear aspects related to the finger competition dynamics [13]. It has been found that competition among the fingering structures is dramatically dependent on the viscosity difference (or viscosity contrast $A$ ) between the fluids. It turns out that changes in the magnitude and sign of $A$ result in fingering patterns presenting very different typical lengths and widths $[2,10,12,13]$. Subsequent numerical studies of the problem $[14,19]$ have verified that the emergence and location of interfacial singularities (pinch-off events) are significantly modified as $A$ varies. These simulations also have substantiated the analytical results of Ref. [13], providing convincing evidence of the usefulness and validity of their weakly nonlinear approach. Similar type of studies including the effect of Coriolis forces still need to be addressed.

In this work, we carry out an analytical weakly nonlinear analysis for the intermediate stages of the interface evolution in rotating Hele-Shaw cells, where the Coriolis effect is significant. We explore the onset of nonlinear effects and try to gain analytical insight into the dynamic process of fingering formation. In contrast to prior work $[1,15,17,18]$ on the un- 


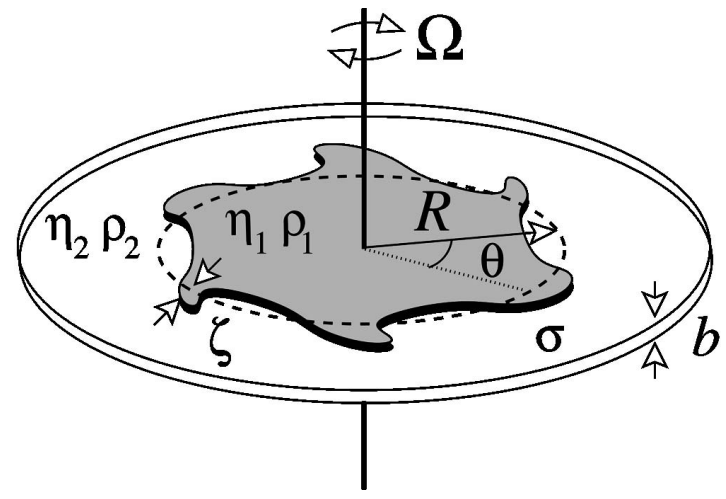

FIG. 1. Schematic diagram representing a rotating Hele-Shaw cell.

bounded outer fluid problem, our study imposes no restrictions on the viscosity contrast, so that $A$ can be arbitrarily taken within its entire range of validity $(-1 \leqslant A \leqslant+1)$. This enables us to extract valuable analytical information concerning the interplay between Coriolis and viscosity contrast effects in both the linear and early nonlinear regimes. We will be particularly interested in studying the impact of these effects on the linear growth rate of interfacial perturbations and on the finger competition dynamics. Comparison of our main results with previous theoretical studies on rotating cells with Coriolis effects $[1,15]$ is also performed.

\section{PHYSICAL PROBLEM AND GOVERNING EQUATIONS}

Consider a rotating Hele-Shaw cell of gap width $b$ containing two immiscible, incompressible, viscous fluids spinning with constant angular velocity $\Omega$ around the $z$ axis, which is perpendicular to (and coaxial with) the cell plates (see Fig. 1). We define our rotating coordinate system in such a way that its origin is located at the center of the cell. The viscosities of the inner and outer fluids are, respectively, denoted as $\eta_{1}$ and $\eta_{2}$, and the surface tension between them is $\sigma$. The densities of the fluids are defined as $\rho_{1}$ and $\rho_{2}$. We consider that the outer fluid is unbounded and focus on the centrifugally induced motion where $\rho_{1}>\rho_{2}$, but allow the inner fluid to be either more or less viscous than the outer fluid. The perturbed interface is described as $\mathcal{R}(\theta, t)=R$ $+\zeta(\theta, t)$ where $R$ is the radius of the initially circular interface and $\zeta(\theta, t)$ represents the net interfacial perturbation in polar coordinates $(r, \theta)$.

Our weakly nonlinear approach follows the theoretical model recently proposed by Waters and Cummings [15] and considers that the fluid flow in the rotating frame of reference is governed the Navier-Stokes equation

$$
\begin{aligned}
\rho_{j}\left[\frac{\partial \mathbf{u}_{j}}{\partial t}+\left(\mathbf{u}_{j} \cdot \nabla\right) \mathbf{u}_{j}\right]= & -\nabla p_{j}+\eta_{j} \nabla^{2} \mathbf{u}_{j}-\rho_{j} \boldsymbol{\Omega} \times(\boldsymbol{\Omega} \times \mathbf{r}) \\
& -2 \rho_{j} \boldsymbol{\Omega} \times \mathbf{u}_{j}
\end{aligned}
$$

and the continuity equation for an incompressible fluid

$$
\boldsymbol{\nabla} \cdot \mathbf{u}_{j}=0,
$$

where $\mathbf{u}_{j}$ denotes the three-dimensional fluid velocity for fluid $j$ (where $j=1,2$ ), $p_{j}$ is the hydrodynamic pressure, $\boldsymbol{\Omega}$ $=\Omega \hat{\mathbf{z}}$ ( $\hat{\mathbf{z}}$ is the unit vector along the $z$ axis), and $\mathbf{r}$ is the position vector of a fluid element measured from the rotation axis. Note that in order to express solutions in the rotating frame of reference at the Hele-Shaw cell, it is necessary to augment the hydrodynamic pressure force balance for motion in an inertial frame with two "fictitious" forces. These are the centrifugal and Coriolis forces, represented by the third and fourth terms on the right-hand side of Eq. (1), respectively. The acceleration due to gravity is neglected.

By using the full Navier-Stokes expression (1), plus Eq. (2), and imposing the no-slip boundary condition at the cell plates $\left(\mathbf{u}_{j}=\mathbf{0}\right.$ at $z=0$ and $\left.z=b\right)$, a generalized Darcy-like law can be obtained by averaging the three-dimensional velocity $\mathbf{u}_{j}$ over the gap direction $(z$ axis)

$$
\mathbf{v}_{j}=-\frac{b^{2} \mathcal{C}_{j}}{12 \eta_{j}}\left[\nabla P_{j}-\frac{\mathcal{D}_{j}}{\mathcal{C}_{j}}\left(\hat{\mathbf{z}} \times \nabla P_{j}\right)\right],
$$

where $\mathbf{v}_{j}(r, \theta)=\int_{0}^{b} \mathbf{u}_{j}(r, \theta, z) d z / b$ represents the 2D gapaveraged velocity in each fluid,

$$
\begin{gathered}
\mathcal{C}_{j}=\frac{\sinh \sqrt{12 \mathrm{Re}_{j}}-\sin \sqrt{12 \mathrm{Re}_{j}}}{2 \operatorname{Re}_{j} \sqrt{12 \mathrm{Re}_{j}}\left[\cosh \sqrt{12 \mathrm{Re}_{j}}+\cos \sqrt{12 \mathrm{Re}_{j}}\right]}, \\
\mathcal{D}_{j}=\frac{1}{2 \operatorname{Re}_{j}}\left\{1-\frac{\sinh \sqrt{12 \mathrm{Re}_{j}}+\sin \sqrt{12 \mathrm{Re}_{j}}}{\sqrt{12 \mathrm{Re}_{j}}\left[\cosh \sqrt{12 \mathrm{Re}_{j}}+\cos \sqrt{12 \mathrm{Re}_{j}}\right]}\right\},
\end{gathered}
$$

with $\operatorname{Re}_{j}=\left(\rho_{j} \Omega b^{2}\right) /\left(12 \eta_{j}\right)$ being a Reynolds number [23]. We stress that in deriving Eq. (3), although the "standard" inertial terms $\left[\partial \mathbf{u}_{j} / \partial t+\left(\mathbf{u}_{j} \cdot \boldsymbol{\nabla}\right) \mathbf{u}_{j}\right]$ appear in the original NavierStokes equation (1), they are later dropped by virtue of the assumed smallness of some reduced Reynolds numbers [15]. The more general, but considerably more complicated case where such inertial terms are retained has been recently examined by Waters et al. in Ref. [16], when the fluids are bounded.

In Eq. (3) the centrifugal contribution is conveniently incorporated into a Laplacian reduced pressure

$$
P_{j}=p_{j}-\frac{\rho_{j} \Omega^{2} r^{2}}{2}
$$

which couples the hydrodynamic pressure $p_{j}$ (which is not Laplacian) to purely centrifugal effects. By using expressions (4) and (5) and expanding to the lowest nonzero order in the Reynolds number we obtain

$$
\mathcal{C}_{j} \approx \frac{1}{1+\alpha \operatorname{Re}_{j}^{2}}, \quad \frac{\mathcal{D}_{j}}{\mathcal{C}_{j}} \approx \beta \mathrm{Re}_{j},
$$

with $\alpha=204 / 35 \approx 5.83$ and $\beta=12 / 5=2.4$. It is worth noting that by taking the zero-Reynolds-number limit $\left(\operatorname{Re}_{j} \rightarrow 0\right)$ of our Eq. (3) we obtain that $\mathcal{C}_{j}=1$ and $\mathcal{D}_{j} / \mathcal{C}_{j}=0$, so that we reproduce the usual Darcy's law equation for a rotating HeleShaw cell without Coriolis forces $[2,10,12,13]$. 
We also call the reader's attention to the fact that in deriving the Darcy-type law, Eq. (3), we have introduced the Coriolis force term already at the level of the Navier-Stokes equation (1). It was under such circumstances that the gapaveraged calculation leading to Eq. (3) has been performed. This was also the case in the work by Waters and Cummings [15]. This procedure is in contrast to Schwartz's work [1] where a simplified Navier-Stokes equation is considered (by neglecting both the "standard" inertial terms and also the Coriolis contribution) and, just after performing its gap averaging, the Coriolis term is put back into the problem in an ad hoc manner. The linear stability analysis performed in Ref. [15] has demonstrated that this arbitrary assumption of Ref. [1] can lead to appreciable errors at the linear level, making it valid only for very small Reynolds numbers. Our mode-coupling theory adopts the more rigorous approach originally proposed in Ref. [15], so this restriction is not imposed on the magnitude of $\operatorname{Re}_{j}$.

We extend the previous linear studies $[1,15]$ to the weakly nonlinear stages of the dynamics and derive the equation of motion for the interfacial perturbations up to second-order couplings. We begin by Fourier expanding the interface perturbation and the corresponding reduced pressure field as

$$
\zeta(\theta, t)=\sum_{n=-\infty}^{+\infty} \zeta_{n}(t) \exp (\operatorname{in} \theta)
$$

with Fourier amplitudes $\zeta_{n}(t)$ and discrete azimuthal wave numbers $n=0, \pm 1, \pm 2, \ldots$, and

$$
P_{j}=\sum_{n \neq 0} P_{j n}(t)\left(\frac{\mathcal{R}^{|n|}}{r^{|n|}}\right)^{(-1)^{j}} \exp (\text { in } \theta),
$$

where expression (8) automatically satisfies Laplace's equation.

We proceed by expressing $P_{j}$ in terms of the perturbation amplitudes $\zeta_{n}$ by considering two basic boundary conditions at the fluid-fluid interface: (i) the kinematic boundary condition $\left.\mathbf{n} \cdot \mathbf{v}_{1}\right|_{\mathcal{R}}=\left.\mathbf{n} \cdot \mathbf{v}_{2}\right|_{\mathcal{R}}$, where $\mathbf{v}_{j}$ is given by Eq. (3) and $\mathbf{n}$ is the unit normal vector pointing from fluid 1 to fluid 2 , and (ii) the pressure jump at the interface $\left.\left(p_{1}-p_{2}\right)\right|_{\mathcal{R}}=\left.\sigma \kappa\right|_{\mathcal{R}}$, where $\kappa$ denotes the interface curvature. By substituting Eqs. (7) and (8) into these boundary conditions, expanding to second order, and Fourier transforming one obtains, after some manipulation, the dimensionless mode-coupling equation for the interfacial amplitudes (for $n \neq 0$ ):

$$
\dot{\zeta}_{n}=\Lambda(n) \zeta_{n}+\sum_{n^{\prime} \neq 0}\left[\mathcal{F}\left(n, n^{\prime}\right) \zeta_{n^{\prime}} \zeta_{n-n^{\prime}}+\mathcal{G}\left(n, n^{\prime}\right) \dot{\zeta}_{n^{\prime}} \zeta_{n-n^{\prime}}\right]
$$

where

$$
\Lambda(n)=\frac{2 \mathcal{E}_{1} \mathcal{E}_{2}}{\left[\left(\mathcal{E}_{1}+\mathcal{E}_{2}\right)+A\left(\mathcal{E}_{1}-\mathcal{E}_{2}\right)\right]}|n|\left[1-B\left(n^{2}-1\right)\right]
$$

is a linear dispersion relation and

$$
\begin{aligned}
\mathcal{F}\left(n, n^{\prime}\right)= & \frac{2 \mathcal{E}_{1} \mathcal{E}_{2}}{\left[\left(\mathcal{E}_{1}+\mathcal{E}_{2}\right)+A\left(\mathcal{E}_{1}-\mathcal{E}_{2}\right)\right]}|n|\left[\frac{1}{2}-B\left(1-\frac{n^{\prime}}{2}\left(3 n^{\prime}\right.\right.\right. \\
& +n))] \\
\mathcal{G}\left(n, n^{\prime}\right)= & {\left[\frac{\left(\mathcal{E}_{1}^{\prime}-\mathcal{E}_{2}^{\prime}\right)+A\left(\mathcal{E}_{1}^{\prime}+\mathcal{E}_{2}^{\prime}\right)}{\left(\mathcal{E}_{1}+\mathcal{E}_{2}\right)+A\left(\mathcal{E}_{1}-\mathcal{E}_{2}\right)}\right]\left[\frac{\mathcal{E}_{1} \mathcal{E}_{2}}{\mathcal{E}_{1}^{\prime} \mathcal{E}_{2}^{\prime}}\right] } \\
& \times\left\{|n|\left[1-\mathcal{H}_{0} \operatorname{sgn}\left(n n^{\prime}\right)\right]-\mathcal{H}_{1}+i\left[\mathcal{I}_{0} \operatorname{sgn}\left(n^{\prime}\right)\right.\right. \\
& \left.\left.+|n| \mathcal{I}_{1} \operatorname{sgn}(n)\right]\right\}
\end{aligned}
$$

represent second-order mode-coupling terms, with $\mathcal{E}_{1}=\mathcal{C}_{1}$ $+i \operatorname{sgn}(n) \mathcal{D}_{1}, \quad \mathcal{E}_{2}=\mathcal{C}_{2}-i \operatorname{sgn}(n) \mathcal{D}_{2}, \quad \mathcal{E}_{1}^{\prime}=\mathcal{C}_{1}+i \operatorname{sgn}\left(n^{\prime}\right) \mathcal{D}_{1}$, and $\mathcal{E}_{2}^{\prime}=\mathcal{C}_{2}-i \operatorname{sgn}\left(n^{\prime}\right) \mathcal{D}_{2}$. The detailed functional form of the terms $\mathcal{H}_{\alpha}$ and $\mathcal{I}_{\alpha}$ (where the index $\alpha=0,1$ ) appearing in Eq. (12) is presented in the Appendix.

Note that all these quantities $(\Lambda, \mathcal{F}$, and $\mathcal{G})$ are complex in general. Perturbations grow (or decay) at a rate $\lambda(n)$ (the linear growth rate), where $\lambda(n)$ is the real part of the complex quantity defined in Eq. (10), $\lambda(n)=\operatorname{Real}[\Lambda(n)]$. The same is true for the mode-coupling terms $F\left(n, n^{\prime}\right)$ $=\operatorname{Real}\left[\mathcal{F}\left(n, n^{\prime}\right)\right]$ and $G\left(n, n^{\prime}\right)=\operatorname{Real}\left[\mathcal{F}\left(n, n^{\prime}\right)\right]$. The sgn function equals \pm 1 according to the sign of its argument. In Eq. (9) lengths are rescaled by $R$ and time by $R / U$, where $U$ $=\left[b^{2} R\left(\rho_{1}-\rho_{2}\right) \Omega^{2}\right] /\left[12\left(\eta_{1}+\eta_{2}\right)\right]$ is a characteristic velocity. Here we define the surface tension parameter $B$ $=\sigma /\left[R^{3} \Omega^{2}\left(\rho_{1}-\rho_{2}\right)\right]$ and the viscosity contrast

$$
A=\frac{\eta_{2}-\eta_{1}}{\eta_{2}+\eta_{1}} .
$$

From now on, we work with the dimensionless version of the equations. Notice that Eq. (9) is conveniently written in terms of the dimensionless parameters $A, B, \mathcal{C}_{j}$, and $\mathcal{D}_{j}$ where these last two are functions of the Reynolds numbers in fluids 1 and 2. The presence of the Coriolis force term in Eq. (3) introduces an explicit dependence of the linear growth rate $\lambda(n)$ and also of the mode-coupling terms $F\left(n, n^{\prime}\right)$ and $G\left(n, n^{\prime}\right)$ on $\operatorname{Re}_{j}$ and $A$. The Reynolds numbers work as controlling parameters that regulate the strength of the Coriolis effects, which are absent when $\mathrm{Re}_{j}=0$ (or equivalently when $\mathcal{C}_{j}=1$ and $\mathcal{D}_{j}=0$ ). In fact, when $\operatorname{Re}_{j}=0$, Eqs. (10)-(12) reproduce the results obtained in Ref. [13] for the corresponding problem without Coriolis effects. We also point out that, after appropriate reintroduction of dimensions, our linear dispersion relation (10) agrees with the Waters-Cummings equivalent formula in the case $A=-1$ [15]. Throughout this work, unless otherwise stated, we focus on the situation in which the centrifugally induced instability is maximized, where the inner fluid is much denser than the outer one $\left(\rho_{1} \gg \rho_{2}\right)$, so that we take $\rho_{2}=0$. Under such circumstances the Coriolis effects are associated with $\mathcal{C}=\mathcal{C}(\mathrm{Re})$ and $\mathcal{D}=\mathcal{D}(\mathrm{Re})$, which are functions of the Reynolds number Re of fluid 1 (hereafter we will drop the subscript 1).

We conclude this section by briefly discussing a connection between our linear dispersion relation (10) and the one very recently obtained in Ref. [16]. Waters and co-workers 
[16] derived a linear dispersion relation for the more complicated case where the "standard" inertial terms are retained [Eq. (3.26) in Ref. [16]]. Notice that it is not exactly straightforward to relate this directly to the results of the present work, because in Ref. [16] the two fluids are contained within a bounded disk (where a no-flux condition is applied on the averaged fluid velocity), with dimensionless radius 1 and inner fluid domain radius $R_{0}$. Conversely, our current results neglect the "standard" inertial terms and consider an unbounded outer fluid. In the notation of Ref. [16] a relation can be obtained by considering the limit in which $\mathcal{A}=O(1)$, while $\mathcal{S} \rightarrow \infty$, with the combination $\mathcal{A S}(1-1 / \widetilde{\rho})=O(1)$, where $\mathcal{A}, \mathcal{S}$, and $\widetilde{\rho}$ are a Strouhal number, the ratio of time scales for flow and rotation, and the density ratio, respectively. Such a comparison still requires some rescaling of lengths (by sending the rigid cell boundary off to infinity in Ref. [16], while keeping $R_{0}$ finite) [24]. We have implemented such a limit and have indeed verified a perfect agreement between our Eq. (10) and Eq. (3.26) in Ref. [16]. A more complete study of the nontrivial comparison between our system and the one studied in Ref. [16] goes beyond the scope of the present work and could be investigated elsewhere.

\section{LINEAR AND WEAKLY NONLINEAR DYNAMICS}

\section{A. Linear stage}

In this section we investigate the consequences of the changes introduced by the generalized Darcy's law (3) in both linear and weakly nonlinear stages of the interface evolution. We get started by discussing some noteworthy features of the linear regime. Note that the linear growth rate $\lambda(n)=\operatorname{Real}[\Lambda(n)][$ see Eq. (10)] is written as the product of a prefactor which depends on Re and $A$, by $|n|\left[1-B\left(n^{2}-1\right)\right]$, which incidentally is the linear growth rate for the case without Coriolis effects [13]. An immediate consequence of the existence of such a prefactor is the fact that both the critical mode number $n_{c}=\sqrt{1+1 / B}$ (defined by setting $\lambda(n)=0$ ) and the fastest-growing mode $n_{\max }=n_{c} / \sqrt{3}$, which maximizes $\lambda(n)$, show no dependence on either Re or $A$. As a result, the band of unstable modes (characterized by $n_{c}$ ) and the typical number of fingers at the linear stage (specified by $n_{\max }$ ) are not affected by the Coriolis effects. Despite having an evident influence on the typical number of fingers shown by the patterned structures, the parameter $B$ will not have much influence on the major results presented in this work. It just introduces some rescaling when varied (smaller $B$ tends to increase the overall magnitude of the effects). Throughout this work and without loss of generality, we take the characteristic value $B=2.0 \times 10^{-4}$. It is worth mentioning that the values of the relevant dimensionless parameters used in our current study $(B, A$, and $\mathrm{Re})$ are absolutely consistent with the typical values of the corresponding physical quantities used in existing experiments in rotating Hele-Shaw cells performed by Carillo et al. [2,4,5] and Alvarez-Lacalle, and co-workers $[12,20]$.

Figure 2 depicts the linear growth rate as a function of mode $n$ for $A=-1$ (black curve), $A=0$ (dark gray), and $A=$
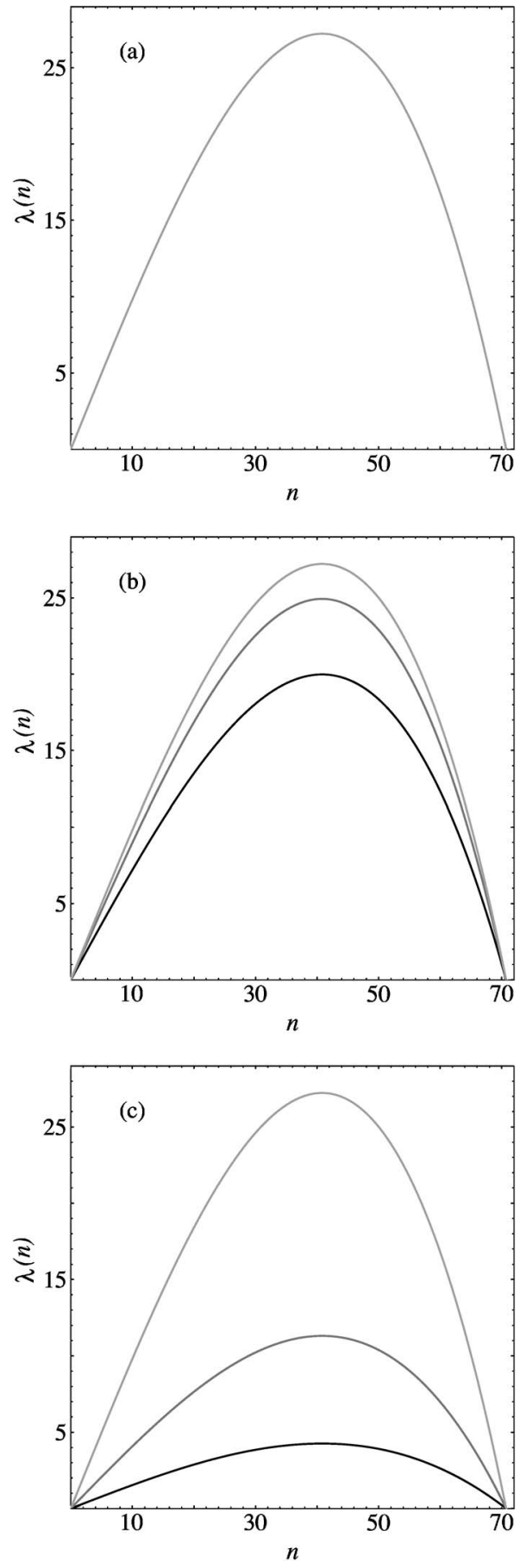

FIG. 2. Linear growth rate $\lambda(n)$ as a function of $n$, for $A=$ $-1,0,+1$ and three different values of Re: (a) 0 , (b) 0.25 , and (c) 1 . The color labeling refers to the values of the viscosity contrast: $A$ $=-1$ (black), $A=0$ (dark gray), and $A=+1$ (light gray). Note that hidden in the solid light gray curve in (a) there are in fact three coincident curves (the other two curves lie hidden).

+1 (light gray) and three different values of Re: (a) 0; (b) 0.25 , and (c) 1 . If Coriolis effects are not included, it can be verified from Eq. (10) that $\lambda(n)$ is completely insensitive to changes in $A$. So in Fig. 2(a) the cases for $A=-1,0,+1$ are all depicted by the same curve. However, if $\operatorname{Re} \neq 0$, the linear growth rate becomes dependent on $A$ and this single curve 


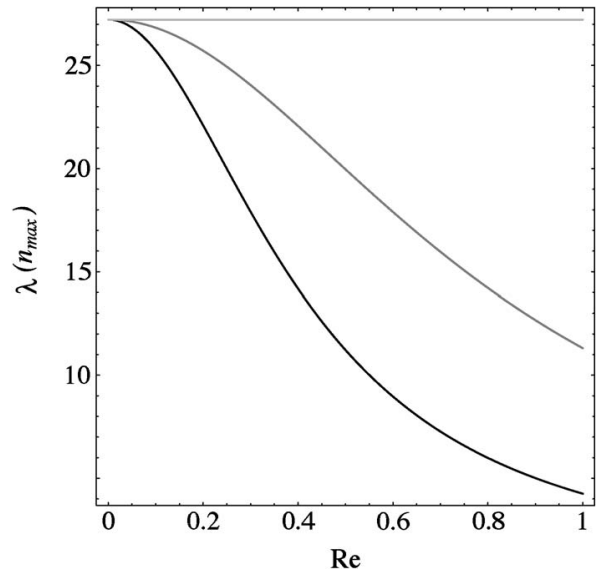

FIG. 3. Maximum growth rate $\lambda\left(n_{\max }\right)$ as a function of Reynolds number Re and $A=-1$ (black), $A=0$ (dark gray), and $A=+1$ (light gray).

splits into others (one for each value of $A$ ) due to Coriolis effects. If $\mathrm{Re}=0.25$ [Fig. 2(b)], we indeed obtain three curves, where the curve for $A=+1 \quad(A=-1)$ has the highest (lowest) peak. So, if $\mathrm{Re}$ is nonzero and is kept fixed while $A$ is modified, the patterns become more unstable as $A$ varies from -1 to +1 . This makes perfect physical sense since when $A=-1$ (inner viscous fluid pushing an outer inviscid fluid) we have the most stable situation regarding the SaffmanTaylor instability. It is also evident from Fig. 2(c) that larger Reynolds numbers $(\mathrm{Re}=1)$ tend to result in curves presenting typically lower peaks for $A \neq+1$, indicating the stabilizing nature of the Coriolis effects. Therefore, if $A$ is kept unchanged and is not equal to +1 , larger Re tend to restrain the growth of interfacial fingering. All these linear stability findings are consistent with recent numerical simulations of the problem $[17,18]$.

By inspecting Eq. (10) it can be verified that Coriolis effects do vanish in the limit of highest positive viscosity contrast $A=+1$, even for nonzero Re. This is the reason why the light gray curve for $A=+1$ depicted in Figs. 2(a)-2(c) remains unchanged as the Reynolds number is modified. Therefore, the zero-Coriolis-force situation can be achieved either by taking $\operatorname{Re}=0$ for any $A$ or by assuming that $A=$ +1 for any $\operatorname{Re} \neq 0$.

It is clearly illustrated in Fig. 2 that despite the stabilizing action of the Coriolis effects, the peak location and width of the band of unstable modes remain unchanged, independently of the values of Re and $A$. A simple physical explanation for this stabilizing role can be given based on the fact that the Coriolis force tends to move the fingers "backwards" in the $\theta$ direction, deviating them from the purely radial growth. Therefore, it inhibits the radial stretching due to centrifugal forces, leading to a decrease in the growth of the interfacial perturbations. Even though the number of fingers is not altered by the Coriolis forces, their radial growth rate is certainly affected.

The aspects highlighted above are also observed in Fig. 3 where the magnitude of the maximum growth rate $\lambda\left(n_{\max }\right)$ is plotted as a function of $0 \leqslant \operatorname{Re} \leqslant 1$ for the same values of $A$ and $B$ used in Fig. 2. If $A=+1$, the height of the peak does not change as Re is varied, meaning that the Coriolis effects play no role. Otherwise, the growth rate of the fastestgrowing mode decreases with increasing Re. This drop of $\lambda\left(n_{\max }\right)$ with $\mathrm{Re}$ is stronger for smaller values of $A$. One important general conclusion is that, in order to induce increasingly larger contributions from the Coriolis force, one needs not merely to consider large values of Re, but also smaller values of $A$. On the other hand, the very presence of $A$ in Eq. (10) comes through the inclusion of Coriolis forces, so that if $\operatorname{Re}=0$, the linear growth rate is completely independent of $A$. These results suggest an interesting coupling between $\mathrm{Re}$ and $A$ when Coriolis effects are taken into account in the rotating Hele-Shaw cell problem.

\section{B. Weakly nonlinear stage}

Now we turn our attention to the weakly nonlinear flow stage, focusing on finger competition events, the prominent nonlinear phenomena occurring in rotating Hele-Shaw cells. It is well known that the viscosity contrast $A$ has a crucial role in determining interfacial behavior for flow in rotating cells when Coriolis effects are neglected [12-14,19]. Considering the length variability as a measure of the finger competition, it has been verified that competition among the fingering structures is dramatically modified as $A$ varies: increasingly larger values of the magnitude of $A>0$ ( $A$ $<0)$ lead to enhanced competition among outward (inward) fingers. It has been also shown that competition is significantly suppressed when $A \rightarrow 0$. When Coriolis effects are significant, in addition to the parameter $A$, it is of interest to examine how finger competition dynamics is affected by the Coriolis control parameter Re.

As in Refs. [13,25], to study finger competition we consider the influence of a fundamental mode $n$ on the growth of its subharmonic $n / 2$. To do that we rewrite the net perturbation (7) in terms of cosine and sine modes, where the cosine $a_{n}=\zeta_{n}+\zeta_{-n}$ and sine $b_{n}=i\left(\zeta_{n}-\zeta_{-n}\right)$ amplitudes are real valued. Then, for consistent second-order expressions, we replace the time derivative terms $\dot{a}_{n}$ and $\dot{b}_{n}$ on the right-hand side of Eq. (9) by $\lambda(n) a_{n}$ and $\lambda(n) b_{n}$, respectively. Without loss of generality we may take $a_{n}>0$ and $b_{n}=0$. Under these circumstances, the following equations of motion for the sine and cosine subharmonics are obtained:

$$
\begin{aligned}
& \dot{a}_{n / 2}=\left\{\lambda(n / 2)+C(n) a_{n}\right\} a_{n / 2}, \\
& \dot{b}_{n / 2}=\left\{\lambda(n / 2)-C(n) a_{n}\right\} b_{n / 2},
\end{aligned}
$$

where

$$
C(n)=\frac{1}{2}\left[F\left(-\frac{n}{2}, \frac{n}{2}\right)+\lambda(n / 2) G\left(\frac{n}{2},-\frac{n}{2}\right)\right] .
$$

The function $C(n)$ measures the strength of the competition such that increasingly larger values of $C(n)>0(C(n)<0)$ lead to enhanced competition of outward (inward) fingers.

We begin our study of the finger competition dynamics by examining the weakly nonlinear results depicted in Fig. 4. It plots $C(n)$ given by Eq. (16) as a function of viscosity contrast $A$ (with $-1 \leqslant A \leqslant 1$ ) for five different values of Rey- 


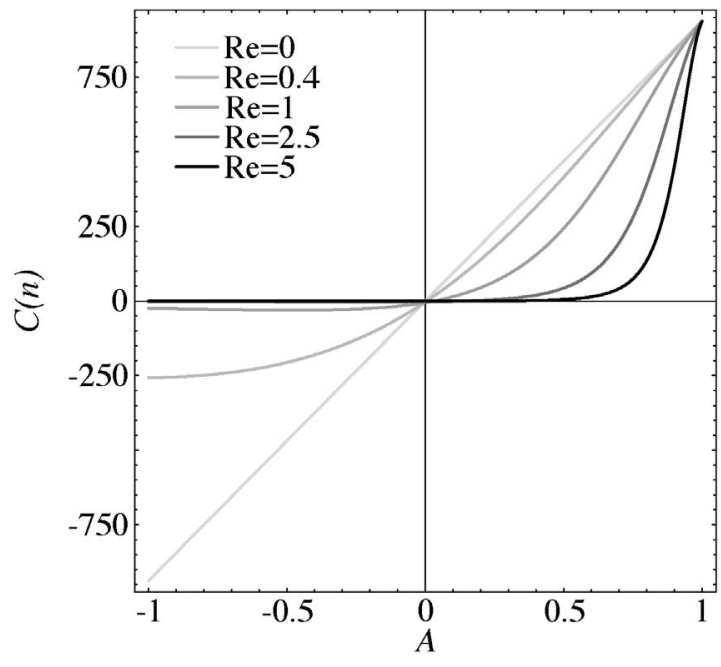

FIG. 4. Competition function $C(n)$ plotted in terms of the viscosity contrast $A$ and five different values of the Reynolds number: $\mathrm{Re}=0,0.4,1,2.5,5$. Darker colors refer to higher Re.

nolds number Re. To observe growth of the fundamental mode and also to allow growth of its subharmonic, we carry out our analysis considering that $n=n_{c}$. The most evident feature of Fig. 4 is the fact that the finger competition behavior is strongly dependent on $\mathrm{Re}$ and can be very different from the equivalent behavior described when Coriolis effects are absent [13]. In Fig. 4, when $\mathrm{Re}=0$ (light gray straight line) we reproduce exactly what has been observed in Refs. [12-14,19] for the case without Coriolis effects.

The situation is significantly changed when the Coriolis effects are taken into account. The first relevant feature when $\operatorname{Re} \neq 0$ is the fact that, in contrast to the case $\operatorname{Re}=0$, the finger competition does not vanish when $A$ tends to zero. Rather, $C(n)=0$ at a specific positive viscosity contrast $\bar{A}$ $=\bar{A}(\mathrm{Re})$. In fact, when Coriolis effects are considered and Re is increased, the competition tends to vanish at increasingly larger values of $\bar{A}$, such that $\bar{A} \rightarrow 1$ for very large values of Re. Consequently, if Coriolis effects are not neglected in rotating Hele-Shaw cells, one could produce a pretty symmetric pattern in which the average length of all fingers is roughly the same, without necessarily imposing that $A=0$. As a matter of fact, depending on the value of Re one could access a situation in which finger competition is practically absent at considerably high $A$. This last conclusion exemplifies how the inclusion of Coriolis effects can introduce significant changes into the nonlinear pattern formation scenario in rotating Hele-Shaw cells.

The competition behavior of the fingers is more complex and richer when Coriolis effects are considered. By examining Fig. 4 and contrasting the case for $\mathrm{Re}=0$ (no Coriolis) to those with $\operatorname{Re} \neq 0$ (nonzero Coriolis effect), we identify some distinct finger competition behaviors. In general, if $A>0$ we notice that the curves for $\operatorname{Re} \neq 0$ lie below the straight line for $\operatorname{Re}=0$, so that a decrease in the finger competition of the outward fingers is expected. On the other hand, if $A<0$ we notice that the curves for $\operatorname{Re} \neq 0$ lie above the straight line for $\operatorname{Re}=0$. Consequently, within this last interval the competition between inward fingers is decreased (even more strongly than when $A>0$ ). The overall effect of the Coriolis forces is to restrain finger competition, regardless of whether they are going inward or outward. We have also verified that for large values of $\mathrm{Re}$, all curves $C(n)$ asymptotically approach zero, except for values of $A$ close to +1 .

\section{PARALLEL WITH SCHWARTZ'S APPROACH}

In this section we make a parallel between our main linear and nonlinear findings (which have been calculated based on the model proposed by Waters and Cummings [15]) with those obtained by using Schwartz's model [1]. By employing Schwartz's approach it is also possible to arrive to a Darcy law equation like the one shown in Eq. (3), however, with different coefficients given by [1]

$$
\mathcal{C}_{j S}=\frac{1}{1+4 \mathrm{Re}_{j}^{2}}, \quad \frac{\mathcal{D}_{j S}}{\mathcal{C}_{j S}}=2 \operatorname{Re}_{j} .
$$

By substituting these coefficients into Eq. (3) and following the same steps described in Sec. II, we obtain a modecoupling differential equation similar to Eq. (9), where the linear growth rate $\lambda(n)_{S}$ and the mode-coupling terms $F\left(n, n^{\prime}\right)_{S}$ and $G\left(n, n^{\prime}\right)_{S}$ for the Schwartz's approach can be directly calculated from Eqs. (10)-(12) simply by replacing $\mathcal{C}_{j}$ by $\mathcal{C}_{j S}$ and $\mathcal{D}_{j}$ by $\mathcal{D}_{j S}$.

First, we compare both growth rates $\lambda(n)$ and $\lambda(n)_{S}$ for the case of interest here $\left(\rho_{2}=0\right)$, as the parameters Re and $A$ are changed. We perform such a comparative study in Fig. 5 which plots the relative percentual error $\Gamma=100\left[\lambda(n)_{S}\right.$ $-\lambda(n)] / \lambda(n)$ as a function of Re for some values of the viscosity contrast. One obvious feature is that, for a given $A$, the error $\Gamma$ undergoes significant changes as the Reynolds number is varied. Of course, if $A=+1$, the growth rates for the two models are coincident and $\Gamma=0$. However, if $-1 \leqslant A<$ +1 , the values of the growth rates coincide only at two specific magnitudes of Re: 0 and $\overline{\operatorname{Re}}=\overline{\operatorname{Re}}(A)$. For a given $A$, the error $\Gamma$ is positive in the interval $0<\operatorname{Re}<\overline{\operatorname{Re}}$ and negative for $\operatorname{Re}>\overline{R e}$. This indicates that Schwartz's approach overestimates the value of the growth rate for the interval $0<\operatorname{Re}$ $<\overline{\mathrm{Re}}$ and underpredicts it for $\mathrm{Re}>\overline{\mathrm{Re}}$. These findings are in agreement with similar results obtained in Ref. [15] for the high-viscosity-contrast case $A=-1$, where $\overline{R e}=2.84$.

It is also evident in Fig. 5 that $\Gamma$ presents a very strong dependence on the viscosity contrast. As $A$ is varied from -1 to +1 , the curves tend to approach the $\Gamma=0$ line, indicating that the value for $\lambda(n)_{S}$ tends to a better agreement with $\lambda(n)$ as $A$ is increased. For the range of Reynolds numbers considered in Fig. $5(0 \leqslant \operatorname{Re} \leqslant 5)$, the largest errors of Schwartz's approach occur precisely at $A=-1(\Gamma \sim 28 \%$ for $\operatorname{Re}=0.92$ and $\Gamma \sim 23 \%$ for $\operatorname{Re}=5$ ). However, these errors tend to decrease as the viscosity contrast varies from -1 to +1 . It is worth noting that the error for underestimation $(\Gamma<0)$ can be much more significant for larger values of Re (for instance, we have verified that $\Gamma$ can be as large as $45 \%$ for $A=-1$ and $\mathrm{Re}=10$ ). We also point out that, for $A=-1$ in Fig. 5 , the error is approximately $5 \%$ for $\mathrm{Re}=0.17$, rising to roughly $10 \%$ around for $\mathrm{Re}=0.25$. This last value of the 


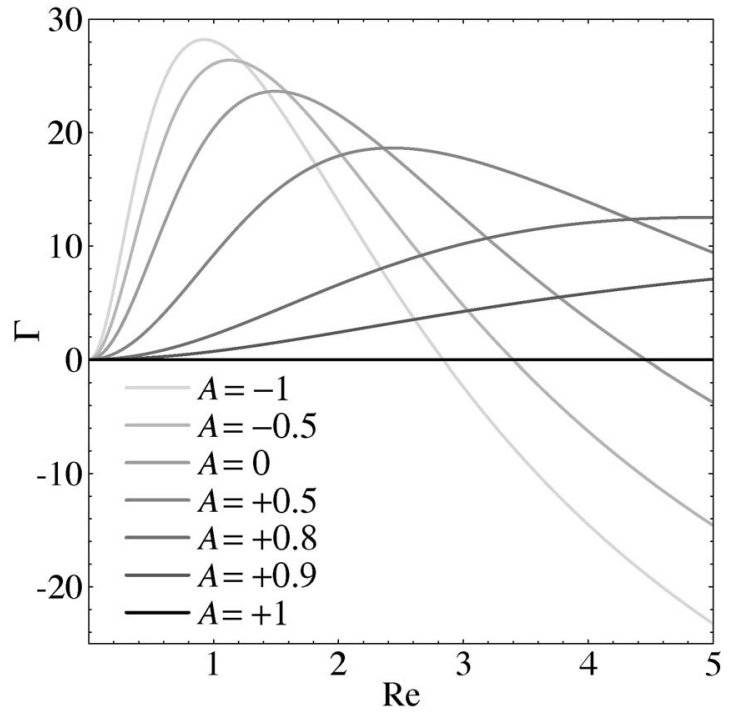

FIG. 5. Percentual error $\Gamma$ as a function of Re, for several values of $A$. Darker colors refer to higher $A$.

Reynolds number has been considered in Ref. [15] (where $A=-1$ ) as the largest value of Re for which the Schwartz's prediction for the linear growth rate would be considered as "good." However, we believe that a 10\% disagreement is a bit too sizable. This indicates that $\Gamma$ can be appreciable already at very small Reynolds numbers.

We proceed by contrasting our approach with Schwartz's at the weakly nonlinear stage. We do this in Figs. 6 and 7. Figure 6 illustrates how the finger competition function behaves as a function of $A$ for three characteristic values of the Reynolds number Re: (a) 0.25, (b) 0.92, and (c) 2.84. The back solid curve refers to $C(n)$ calculated from Eq. (16), and the back dashed one is related the equivalent function $C(n)_{S}$ we derived based on Schwartz's model. Complementary information can be obtained in Fig. 7, where we plot the difference $C(n)_{S}-C(n)$ as a function of $A$ for the same values of Re used in Fig. 6.

As one can easily observe in Fig. 6(a) for low Re, in general the mismatch between the two descriptions is relatively small for most values of the viscosity contrast $A$, but increases significantly as $A \rightarrow-1$. This feature is also clearly illustrated in Fig. 7(a), where the difference between the models is particularly large within the interval $-1 \leqslant A<0$. As far as finger competition is concerned, it can be noted that even if $\mathrm{Re}$ is as low as 0.25 the difference between the two approaches at the weakly nonlinear level can still be pretty significant, depending on the value of $A$.

A different scenario is depicted in Figs. 6(b) and 7(b) when $\operatorname{Re}=0.92$ : now the discrepancies between the two approaches are significantly larger for $A>0$ (relative to what happens for the case $\mathrm{Re}=0.25$ ) and are indeed present along the whole range of values for the viscosity contrast $(-1 \leqslant A$ $<+1$ ). It is also noticed in Fig. 6(b) that as $A$ is varied the dashed curve can be located above or below the solid one, indicating that Schwartz's model can be misleading regarding the finger competition dynamics. This behavior is even more clear in Fig. 7(b). As the Reynolds number is further increased as in Figs. 6(c) and 7(c) in which $\mathrm{Re}=2.84$ we
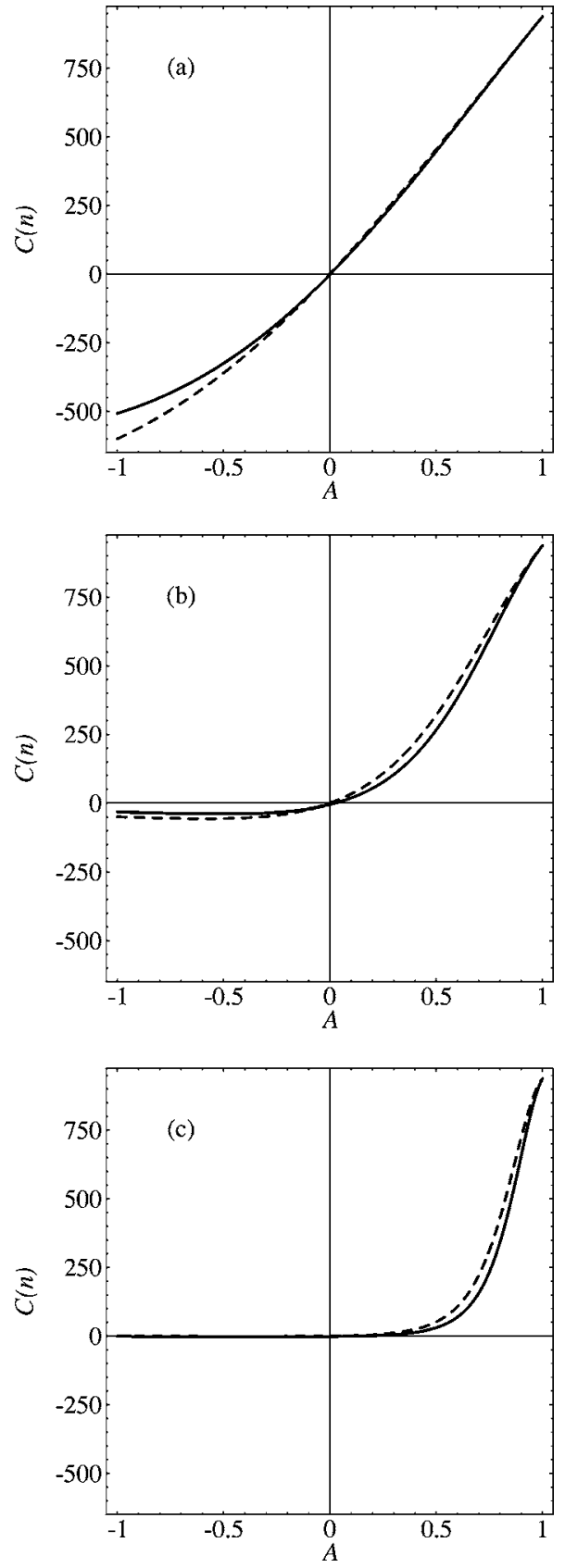

FIG. 6. Competition functions $C(n)$ (black solid curve) and $C(n)_{S}$ (black dashed curve) as a function of $A$ for three different values of Re: (a) 0.25 , (b) 0.92 , and (c) 2.84 .

notice a behavior that is kind of the inverse of the one shown in Figs. 6(a) and 7(a): now the disagreements between the models are very small for $-1<A<0$, but notably increased within the interval $0<A<+1$.

By inspecting Figs. 6 and 7 it is apparent that, for a given $\mathrm{Re}$, the difference between the two approaches is quite dependent on the value of the viscosity contrast. It is also interesting to note that the regions of the largest discrepancy migrate from values around $A=-1$ for lower Reynolds number to the vicinity of $A=+1$ for larger Re. On the other hand, regardless the value of Re there exist some values of $A$ in which there is no difference between the two approaches 


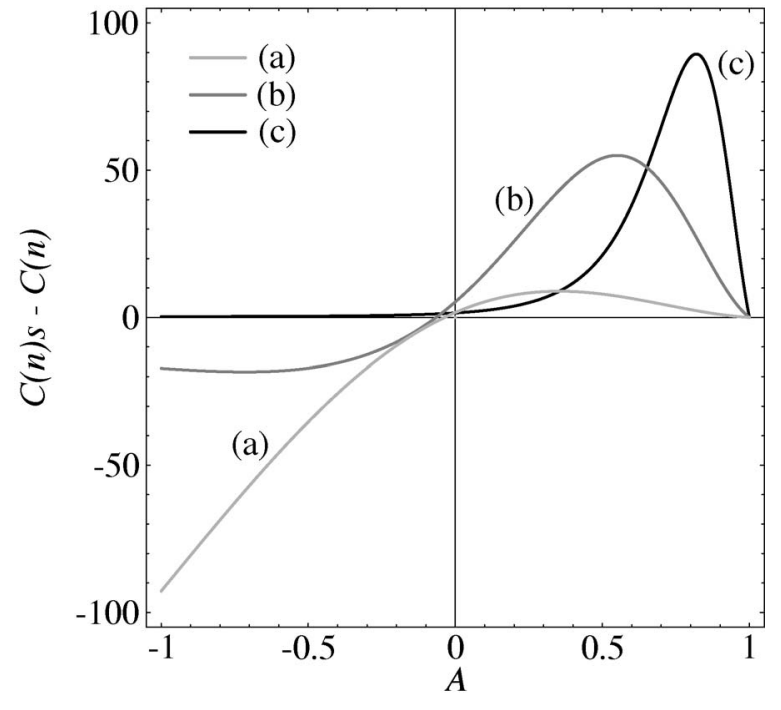

FIG. 7. Difference $C(n)_{S}-C(n)$ as a function of $A$ for three different values of Re: (a) 0.25, (b) 0.92, and (c) 2.84. Darker colors refer to higher Re.

[given by the zero of $C(n)_{S}-C(n)$ ]. All these results indicate that Schwartz's approach may result in significant errors at weakly nonlinear stages.

\section{CONCLUSIONS}

We have investigated the effect of Coriolis force on the flow and interfacial pattern formation in rotating Hele-Shaw cells. The system is described by a generalized 2D Darcy's law, derived by a gap-averaging process of the 3D NavierStokes equation properly including Coriolis effects. We carried out a mode-coupling approach for the system and derived an equation of motion for the interfacial perturbations up to second order. This equation is conveniently written in terms of the viscosity contrast $A$ and the Reynolds numbers of the fluids.

Our analytical study extends the previous contributions of the problem $[1,15,17,18]$ in several directions. First, the viscosity contrast may assume arbitrary values. This allowed us to reveal the interplay between $A$ and Re in determining the action of Coriolis effects on the behavior of the evolving fluid-fluid interface. Some noteworthy behaviors can be already identified at the linear level, including the stabilizing nature of the Coriolis force and the mentioned connection between Re and $A$ when such forces are present. We have shown that the magnitude of the Coriolis effects is not exclusively dependent on the intensity of the Reynolds number. Consequently, stronger Coriolis effects are achieved not only by setting high values of Re, but also by taking smaller values of $A$. We have also verified that when $\rho_{2}=0$, regardless the value of $\mathrm{Re}_{1}$, the Coriolis effects completely vanish if $A=+1$.

In addition, our study goes beyond the purely linear stages of the dynamics and addresses relevant nonlinear aspects related to the finger competition phenomena. One interesting finding was the fact that, in contrast to the rotating flow case without Coriolis effects, if $\operatorname{Re} \neq 0$, the finger competition does not vanish when $A=0$. In fact, we have found that if Coriolis effects are significant, competition is absent only for relatively large values of the viscosity contrast. We have also been able to contrast our main results with similar ones we obtained from the simplified Coriolis approach proposed sometime ago by Schwartz [1]. We have demonstrated that the neglect of Coriolis effects (at the level of the 3D NavierStokes equation) in deriving a 2D Darcy's law for the problem can lead to significant errors at both linear and weakly nonlinear levels.

In a more general sense, our work is related to some other recent efforts that try to introduce inertial corrections into the Saffman-Taylor problem [26-29]. These studies and our own are necessary to provide a better understanding of the emerging fingering instabilities for the high-Reynolds-number limit. In our case the inertial corrections associated with the Coriolis force can be included into a Darcy's-law-type equation in a relatively simple fashion, a fact that greatly facilitated the study of Coriolis effects at both linear and nonlinear levels. We hope our study will be useful in the investigation of other pattern-forming systems involving similar swirling effects, where spiral patterns arise in magnetic fluids [30-34] and lipid monolayer domains [35].

\section{ACKNOWLEDGMENTS}

We would like to thank the Brazilian Research CouncilCNPq (J.A.M. and H.G.) and FACEPE (N.B.) for financial support of this research. J.A.M. also thanks CNPq for support through the PDE Proceeding No. 200045/2005-9 and program "Instituto do Milênio de Fluidos Complexos" Contract No. 420082/2005-0. We gratefully acknowledge useful communications and stimulating discussions with Linda Cummings and Sarah Waters.

\section{APPENDIX: FUNCTIONS $\mathcal{H}_{\alpha}$ and $\mathcal{I}_{\alpha}$}

This short appendix presents the complicated expressions for the functions $\mathcal{H}_{\alpha}$ and $\mathcal{I}_{\alpha}$ appearing in the second-order mode-coupling coefficient $G\left(n, n^{\prime}\right)$ in Eq. (12):

$$
\begin{gathered}
\mathcal{H}_{\alpha}=\frac{\left[A^{1-\alpha}\left(\frac{\mathcal{E}_{2}^{\prime}}{\mathcal{E}_{1}} \mathcal{C}_{1}+\frac{\mathcal{E}_{1}^{\prime}}{\mathcal{E}_{2}} \mathcal{C}_{2}\right)-A^{\alpha}\left(\frac{\mathcal{E}_{2}^{\prime}}{\mathcal{E}_{1}} \mathcal{C}_{1}-\frac{\mathcal{E}_{1}^{\prime}}{\mathcal{E}_{2}} \mathcal{C}_{2}\right)\right]}{f\left(\mathcal{E}_{1}^{\prime}, \mathcal{E}_{2}^{\prime}, A\right)}, \\
\mathcal{I}_{\alpha}=\frac{\left[A^{1-\alpha}\left(\frac{\mathcal{E}_{2}^{\prime}}{\mathcal{E}_{1}} \mathcal{D}_{1}+\frac{\mathcal{E}_{1}^{\prime}}{\mathcal{E}_{2}} \mathcal{D}_{2}\right)-A^{\alpha}\left(\frac{\mathcal{E}_{2}^{\prime}}{\mathcal{E}_{1}} \mathcal{D}_{1}-\frac{\mathcal{E}_{1}^{\prime}}{\mathcal{E}_{2}} \mathcal{D}_{2}\right)\right]}{f\left(\mathcal{E}_{1}^{\prime}, \mathcal{E}_{2}^{\prime}, A\right)},
\end{gathered}
$$

where $f\left(\mathcal{E}_{1}^{\prime}, \mathcal{E}_{2}^{\prime}, A\right)=\left[\left(\mathcal{E}_{1}^{\prime}-\mathcal{E}_{2}^{\prime}\right)+A\left(\mathcal{E}_{1}^{\prime}+\mathcal{E}_{2}^{\prime}\right)\right]$ and $\alpha=0,1$. 
[1] L. W. Schwartz, Phys. Fluids A 1, 167 (1989).

[2] Ll. Carrillo, F. X. Magdaleno, J. Casademunt, and J. Ortín, Phys. Rev. E 54, 6260 (1996).

[3] V. M. Entov, P. I. Etingof, and D. Ya. Kleinbock, Eur. J. Appl. Math. 6, 399 (1996).

[4] Ll. Carrillo, J. Soriano, and J. Ortín, Phys. Fluids 11, 778 (1999).

[5] Ll. Carrillo, J. Soriano, and J. Ortín, Phys. Fluids 12, 1685 (2000).

[6] F. X. Magdaleno, A. Rocco, and J. Casademunt, Phys. Rev. E 62, R5887 (2000).

[7] J. A. Miranda, Phys. Rev. E 62, 2985 (2000).

[8] C.-Y. Chen and S.-W. Wang, Fluid Dyn. Res. 30, 315 (2002).

[9] D. Crowdy, Q. Appl. Math. 60, 11 (2002); SIAM J. Appl. Math. 62, 945 (2002).

[10] E. Alvarez-Lacalle, E. Pauné, J. Casademunt, and J. Ortín, Phys. Rev. E 68, 026308 (2003).

[11] D. P. Jackson and J. A. Miranda, Phys. Rev. E 67, 017301 (2003).

[12] E. Alvarez-Lacalle, J. Ortín, and J. Casademunt, Phys. Fluids 16, 908 (2004).

[13] H. Gadêlha and J. A. Miranda, Phys. Rev. E 70, 066308 (2004).

[14] J. A. Miranda, and E. Alvarez-Lacalle, Phys. Rev. E 72, 026306 (2005).

[15] S. L. Waters and L. J. Cummings, Phys. Fluids 17, 048101 (2005).

[16] S. L. Waters, L. J. Cummings, K. M. Shakesheff, and F. R. A. J. Rose, IMA J. Math. Appl. Med. Biol. 23, 311 (2006).

[17] C.-Y. Chen and Y.-C. Liu, Int. J. Numer. Methods Fluids 48, 853 (2005).

[18] C.-H. Chen and C.-Y. Chen, Int. J. Numer. Methods Fluids 51,
881 (2006).

[19] C.-Y. Chen, C.-H. Chen, and J. A. Miranda, Phys. Rev. E 73, 046306 (2006).

[20] E. Alvarez-Lacalle, J. Ortín, and J. Casademunt, Phys. Rev. E 74, 025302(R) (2006).

[21] P. G. Saffman and G. I. Taylor, Proc. R. Soc. London, Ser. A 245, 312 (1958).

[22] L. Paterson, J. Fluid Mech. 113, 513 (1981).

[23] Even though we keep the same notation used in Ref. [15] for the terms $\mathcal{C}_{j}$ and $\mathcal{D}_{j}$, we point out that the magnitudes of our terms in Eqs. (4) and (5) are 12 times larger than the ones defined in Ref. [15].

[24] This suggestive limit has been pointed out to us by L. J. Cummings and S. L. Waters, (private communication) as a possible starting point to connect our linear stability results.

[25] J. A. Miranda and M. Widom, Physica D 120, 315 (1998).

[26] P. Gondret and M. Rabaud, Phys. Fluids 9, 3267 (1997).

[27] C. Ruyer-Quil, C. R. Acad. Sci., Ser. IIb: Mec., Phys., Chim., Astron. 329, 337 (2001).

[28] F. Plouraboue and E. J. Hinch, Phys. Fluids 14, 922 (2002).

[29] C. Chevalier, M. Ben Amar, D. Bonn, and A. Lindner, J. Fluid Mech. 552, 83 (2006).

[30] J.-C. Bacri, A. Cebers, and R. Perzynski, Phys. Rev. Lett. 72, 2705 (1994).

[31] S. Lacis, J. Magn. Magn. Mater. 201, 335 (1999).

[32] C. Lorenz and M. Zahn, Phys. Fluids 15, S4 (2003).

[33] S. Rhodes, J. Perez, S. Elborai, S.-H. Lee, and M. Zahn, J. Magn. Magn. Mater. 289, 353 (2005).

[34] S. Elborai, D.-K. Kim, X. He, S.-H. Lee, S. Rhodes, and M. Zahn, J. Appl. Phys. 97, 10Q302 (2005).

[35] P. Krüger and M. Lösche, Phys. Rev. E 62, 7031 (2000). 